\title{
X-ray Polarization from Accreting Black Holes: II. The Thermal State
}

\author{
Jeremy D. Schnittman \\ Department of Physics and Astronomy, Johns Hopkins University \\ Baltimore, MD 21218 \\ schnittm@pha.jhu.edu \\ and \\ Julian H. Krolik \\ Department of Physics and Astronomy, Johns Hopkins University \\ Baltimore, MD 21218 \\ jhk@pha.jhu.edu
}

\begin{abstract}
We present new calculations of X-ray polarization from black hole (BH) accretion disks in the thermally-dominated state, using a Monte-Carlo ray-tracing code in full general relativity. In contrast to many previously published studies, our approach allows us to include returning radiation that is deflected by the strong-field gravity of the $\mathrm{BH}$ and scatters off of the disk before reaching a distant observer. Although carrying a relatively small fraction of the total observed flux, the scattered radiation tends to be highly polarized and in a direction perpendicular to the direct radiation. For moderately large spin parameters $(a / M \gtrsim 0.9)$, this scattered returning radiation dominates the polarization signal at energies above the thermal peak, giving a net rotation in the polarization angle of $90^{\circ}$. We show how these new features of the polarization spectra from BHs in the thermal state may be developed into a powerful tool for measuring $\mathrm{BH}$ spin and probing the gas flow in the innermost disk. In addition to determining the emission profile, polarization observations can be used to constrain other properties of the system such as BH mass, inclination, and distance. New instruments currently under development should be able to exploit this tool in the near future.
\end{abstract}

Subject headings: black hole physics - accretion disks - X-rays:binaries 


\section{INTRODUCTION}

A recent flurry of new mission proposals has renewed interest in X-ray polarization from a variety of astrophysical sources. The Gravity and Extreme Magnetism SMEX (GEMS) mission 1 , which has recently been approved for phase A study in the latest round of NASA Small Explorer proposals, should be able to detect a degree of polarization $\delta \lesssim 1 \%$ for a flux of a few mCrab (Black et al. 2003; Bellazzini et al. 2006). A similar detector for the International X-ray Observatory $(I X O)$ could achieve sensitivity roughly $10 \times$ greater $(\lesssim 0.1 \%$ degree of polarization; Jahoda et al. 2007, Costa et al. 2008). A large number of galactic and extra-galactic sources are expected to be polarized at the $\delta \gtrsim 1 \%$ level, including black holes, magnetars, pulsar wind nebulae, and active galactic nuclei. In this paper, we focus on accreting stellar-mass black holes $(\mathrm{BHs})$ in the thermal state, which are characterized by a broad-band spectrum peaking around $1 \mathrm{keV}$. The typical level of polarization from these sources should be a few percent in the $1-10 \mathrm{keV}$ range, depending on $\mathrm{BH}$ spin and the inclination angle of the accretion disk.

Symmetry arguments demand that in the flat-space (Newtonian) limit, the observed polarization from the disk must be either parallel or perpendicular to the $\mathrm{BH} /$ disk rotation axis. However, the effects of relativistic beaming, gravitational lensing, and gravito-magnetic frame-dragging can combine to give a non-trivial net rotation to the integrated polarization vector. Early work exploring these effects (Stark \& Connors 1977; Connors \& Stark 1977; Connors et al. 1980) showed that they create changes in the angle and degree of polarization that are strongest for higher photon energy. The reason for this trend is that the temperature in a standard accretion disk increases as the BH is approached, and that is, of course, where the relativistic effects are strongest. Since those first efforts, there have been studies of the predicted polarization from more general accretion geometries, including UV and X-ray emission from AGN disks (Laor et al. 1990; Matt et al. 1993) and "lamp post" models for irradiating the accretion disk with a non-thermal source above the plane (Dovciak et al. 2004). Quite recently, Dovciak et al. (2008) investigated the effect of atmospheric optical depth on the polarization signal, and Li et al. (2008) applied the original calculations of thermal X-ray polarization to the problem of measuring the inclination of the inner accretion disk.

Despite this relatively large body of literature dedicated to X-ray polarization from $\mathrm{BH}$ accretion disks, to the best of our knowledge, all previous work has modeled the relativistic effects by calculating the transfer function along geodesics between the observer and emitter. By its very nature, this method precludes the possibility of including the effects of returning

\footnotetext{
${ }^{1}$ heasarc.gsfc.nasa.gov/docs/gems
} 
radiation-photons emitted from one part of the disk and bent by gravitational lensing so that they are absorbed or reflected from another part of the disk (Cunningham 1976). Using new methodology, in this paper we show the contribution from this returning radiation is very important to the observed polarization. The details of our method are described in Schnittman (2009) (hereafter "Paper I"). The most important feature of our approach is that the photons are traced from the emitting region in all directions, either returning to the disk, scattering through a corona, getting captured by the $\mathrm{BH}$, or eventually reaching a distant observer. It should be noted that a sort of hybrid technique was used in Agol \& Krolik (2000) to estimate the effects of returning radiation on polarization by situating an observer in the disk plane and shooting rays backwards to see how much flux was incident from other regions of the disk, but this approach cannot account for multiple scattering events, where the returning radiation passes over the $\mathrm{BH}$ more than once.

The inclusion of returning radiation leads to the most important of the new results presented in this paper, namely a transition between horizontal- and vertical-oriented polarization as the photon energy increases. At low energies we reproduce the "Newtonian" result of a semi-infinite scattering atmosphere emitting radiation weakly polarized in a direction parallel to the emission surface, an orientation we call horizontal polarization (Chandrasekhar 1960). At higher energy, corresponding to the higher temperature of the inner disk, a greater fraction of the emitted photons returns to the disk and is then scattered to the observer. These scattered photons have a high degree of polarization and are aligned parallel to the disk rotation axis (vertical), as projected onto the image plane. At the transition point between horizontal and vertical polarization, the relative contributions of direct and reflected photons are nearly equal, and no net polarization is observed.

Since the effects of returning radiation are greatest for photons coming from the innermost regions of the disk, the predicted polarization signature is strongly dependent on the behavior of gas near and inside the inner-most stable circular orbit (ISCO). Thus, polarization observations could be used to measure the spin of the black hole and also constrain the dissipation profile of the inner disk. For systems where the BH mass, distance, and disk inclination are known, a single polarization observation can constrain the emission profile at least well as fitting the thermal continuum spectrum (Gierliński et al. 2001; Davis et al. 2006; Shafee et al. 2006). When these system parameters are not known a priori, polarization can be used in many cases to measure them simultaneously with the emission profile (albeit with lower confidence than for the sources with known priors).

The structure of this paper is as follows: after briefly describing our methods in Section 2. we review in Section 3 some of the classical results of earlier polarization calculations from thermal disks, including only the radiation that reaches the observer directly from the disk. 
In Section 4, we include the effects of returning radiation, focusing on new features in the polarization spectrum. Sections 5 and 6 contain a detailed discussion of our results and how they depend on model parameters, and in Section 7 we conclude.

\section{METHODOLOGY}

The inner disk of a stellar-mass BH binary can be well described locally as a planeparallel, electron scattering-dominated atmosphere (Shakura \& Sunvaev 1973; Novikov \& Thorne 1973). In the local fluid frame, the classical result of Chandrasekhar (1960) applies: the polarization vector of the emitted radiation is oriented parallel to the disk plane and normal to the direction of propagation. The degree of polarization varies from zero for photons emitted normal to the disk surface up to $\sim 12 \%$ for an inclination angle of $90^{\circ}$. In addition to the polarization effects, the scattering of the outgoing flux causes limb darkening, effectively focusing the emitted radiation in the direction normal to the disk surface. Both the degree of polarization and the limb darkening/brightening factors are tabulated as a function of emission angle in Table XXIV in Chandrasekhar (1960).

For the local emission spectrum, we assume a diluted black-body spectrum with hardening factor $f=1.8$ (Shimura \& Takahara 1995). As demonstrated by Davis et al. (2005), this is a reasonable approximation for disks around stellar-mass black holes because the temperature is so high that there is little opacity due to anything but Compton scattering and free-free absorption. For a locally emitted flux $\mathcal{F}$, the effective temperature is defined as

$$
T_{\text {eff }} \equiv\left(\frac{\mathcal{F}}{\sigma}\right)^{1 / 4}
$$

where $\sigma$ is the Stefan-Boltzmann constant. The spectral intensity of the diluted emission is then given by

$$
I_{\nu}=\frac{1}{f^{4}} B_{\nu}\left(f T_{\mathrm{eff}}\right)
$$

with $B_{\nu}$ the black-body function. For the emitted flux function $\mathcal{F}(R)$, we consider two different models: that given by Novikov \& Thorne (1973) for a zero-stress inner boundary condition at the ISCO, and a quasi-Newtonian expression $\mathcal{F}(R) \sim R^{-3}$ that extends the classical scaling at large radius all the way down to the horizon. Recent global magnetohydrodynamic (MHD) simulations suggest that reality lies somewhere in between these two models (Noble et al. 2008). Outside of the ISCO, the gas follows circular, planar geodesic

orbits as in Novikov \& Thorne (1973). Inside the ISCO, the fluid continues along plunging trajectories with constant energy and angular momentum, resulting in a rapid decline of surface density with decreasing radius. 
As described in Paper I, at each point in the disk, multi-wavelength photon packets are emitted in all directions, with appropriate intensity weights and polarizations as a function of emission angle. We use a Monte Carlo algorithm with typically $10^{5}-10^{6}$ rays traced per radius, with 500 radial bins spaced logarithmically between the horizon and $R=100 M$. Since the photon trajectories originate at the disk, the observer's inclination angle is not specified; rather, all photons are followed until they get captured by the BH, return to the disk plane, or reach "infinity," in practice a spherical hypersurface at large radius. At that point, the photons are binned by observer inclination, effectively simulating all inclinations simultaneously. By calculating the angle that each photon makes with the detector sphere, we are also able to create an image of the source, much like a classical pin-hole camera.

Each photon packet contains a complete broad-band spectrum initially described by equation (2). Since Thomson scattering in the disk atmosphere is frequency-independent over the range of relevant X-ray energies $(\sim 0.1-10 \mathrm{keV}$ for thermal emission from a solar-mass BH), the photon packet can be described by a single polarization amplitude and direction.

Following Connors et al. (1980), we characterize the polarization of each photon packet by the normalized Stokes parameters:

$$
\begin{aligned}
& X=Q / I, \\
& Y=U / I
\end{aligned}
$$

and

$$
\begin{aligned}
\delta & =\left(X^{2}+Y^{2}\right)^{1 / 2}, \\
\psi & =\frac{1}{2} \tan ^{-1}(Y / X),
\end{aligned}
$$

where $\psi$ is the angle of polarization, ranging from $-90^{\circ}$ to $+90^{\circ}$, and $\delta \leq 1$ is the degree of polarization, invariant along a geodesic.

The polarization vector $\mathbf{f}$ is a normalized space-like 4-vector perpendicular to the photon propagation direction: $\mathbf{k} \cdot \mathbf{f}=0$ and $\mathbf{f} \cdot \mathbf{f}=1$. It is parallel-transported along the photon's null geodesic, conserving the complex-valued Walker-Penrose integral of motion $\kappa$ (Walker \& Penrose 1970). Analogous to the way that Carter's constant (Carter 1968) can be used to constrain four-velocity components, $\kappa$ can be used to reconstruct the polarization vector at any point along the geodesic. The two orthonormality conditions stated above, along with the real and imaginary parts of $\kappa$, give a total of four equations for the four components of the polarization vector $\mathbf{f}$. When a ray reaches an observer at infinity, the polarization vector is projected onto the detector plane defined by the basis vectors $\mathbf{e}_{1}$ and $\mathbf{e}_{2}$ to 
give the polarization angle: $\mathbf{f}=\left[0, \cos \psi \mathbf{e}_{1}+\sin \psi \mathbf{e}_{2}\right]$ (in our convention, $\mathbf{e}_{2}$ is parallel to the projected rotation axis of the $\mathrm{BH}$ and disk). From $\delta$ and $\psi$, as measured by the detector, we can reconstruct the normalized Stokes parameters $X$ and $Y$ via equation (4). The observed spectrum $I_{\nu}$ is propagated along the geodesic path as well, containing within it the emitted spectrum, modified by all relativistic effects as well as any energy change that may occur when returning radiation scatters off disk electrons. $I_{\nu}$ provides the absolute normalization that, when applied to $X$ and $Y$ allows $Q_{\nu}$ and $U_{\nu}$ to be recovered for each photon bundle reaching the detector. Integrating over all photon bundles gives the polarization degree $\delta_{\nu}$ and angle $\psi_{\nu}$ as a function of energy (see Paper I for details). These are ultimately the quantities that we wish to be able to measure and use to probe the accretion geometry of the $\mathrm{BH}$.

\section{DIRECT RADIATION}

In Figure 1, we show a simulated image of a Novikov-Thorne accretion disk around a black hole with spin parameter $a / M=0.9$ and luminosity $0.1 L_{E}$, corresponding to a disk whose X-ray spectrum peaks around $1 \mathrm{keV}$ for a $\mathrm{BH}$ mass of $10 M_{\odot}$. With the observer at an inclination of $i=75^{\circ}$, significant relativistic effects are clearly apparent. The increased intensity on the left side of the disk is due to special relativistic beaming of the gas moving towards the observer, and the general relativistic light bending makes the far side of the disk appear warped and bent up towards the observer. Superposed on top of the intensity map is the polarization signature, represented by small black vectors whose lengths are proportional to the degree of polarization observed from that local patch of the disk. Far from the black hole, the polarization is essentially given by the classical result of Chandrasekhar (1960) for a scattering-dominated atmosphere: horizontal orientation with $\delta \approx 4 \%$ when $i=75^{\circ}$; nearer to the black hole, a variety of relativistic effects alter the polarization.

While the fundamental calculation of polarization from a thermal $\mathrm{BH}$ accretion disk is not at all new (Connors et al. 1980), to the best of our knowledge this is the first published polarization map as seen projected in the image plane. The usefulness of such a map is primarily as a tool to understand how relativistic effects determine the integrated polarization at different energies.

The two most prominent relativistic effects are gravitational lensing and special relativistic beaming, both lowering the net level of polarization seen by the observer. Gravitational lensing causes the far side of the disk to appear warped up towards the observer, and thus have a smaller effective inclination. Relativistic beaming causes photons emitted normal to the disk plane in the fluid frame to travel forward in the direction of the local orbital motion 
when seen by a distant observer; the result is a smaller effective inclination and thus degree of polarization. On the other hand, photons emitted at high inclination angle in the local fluid frame but against the direction of orbital motion are ultimately seen by observers located at lower inclination, and therefore raise the polarization in that direction. Naturally, these relativistic effects are most important close to the black hole, where the gas is also hottest and the photons have the highest energies. All these effects are clearly visible in Figure 1, which shows a smaller degree of polarization where the beaming is greatest (yellow region of high intensity in the left of the image) and the lensing is strongest (just above the center of the image). At the same time, the gas moving away from the observer on the right of the image has an enhanced level of polarization because the observer sees photons emitted at a larger inclination in the fluid frame.

Integrating over the entire disk, we can calculate the angle and degree of polarization as a function of energy. Plotted in Figure 2, these results essentially reproduce those of Connors et al. (1980), who performed a similar calculation using semi-analytic transfer functions from the observer to the disk. Again we see that at low energies (dominated by emission from large disk radii), the polarization is given by the Chandrasekhar result, and is a function only of the disk inclination angle. At higher energies, we begin to probe the inner regions of the accretion disk and see the relativistic effects described above that lower the total degree of polarization. In addition to reducing the polarization amplitude, the strong gravity near the black hole also rotates the angle of polarization due to the parallel transport: since the polarization vector $\mathbf{f}$ must remain perpendicular to the photon momentum $\mathbf{k}$, as the geodesic path bends around the $\mathrm{BH}$, the polarization angle must also rotate. For example, the high-energy photons beamed towards the observer in Figure 1 are also bent by the BH's gravity, initially moving to the left before curving back to the observer, thus producing the rotated polarization vectors seen just to the left of the image center (the same lensing effect rotates the red-shifted photons from the right side of the disk, but in an opposite direction).

The angle of polarization as a function of energy is plotted in the lower panel of Figure 2. For an inclined accretion disk with the black hole axis projected onto the vertical, or y-axis, $\psi=0$ corresponds to horizontal polarization parallel to the disk surface. From equation (4), we see there is a point symmetry in the definition of $\psi$, giving $\psi=\psi \pm 180^{\circ}$. As can be seen in the simulated image of Figure1, the individual polarization vectors are rotated in the inner disk, due largely to gravitational lensing and, to a lesser degree, frame-dragging around a spinning black hole. While individual photons can experience significant rotation, the net result is a modest rotation of the total observed polarization in the clockwise direction, giving $\psi<0$ at higher energies. From Figure 2, it is clear this effect is more pronounced for small inclinations, where the low amplitude of polarization makes it easier to "overcome" the classical result with additional relativistic effects. Similarly, the polarization rotation 
is greater for more rapidly spinning black holes, where the accretion disk extends closer in towards the horizon and probes a stronger gravitational field. In this way, it has previously been proposed that the polarization degree and angle as a function of energy could be used to infer the spin of the black hole in the thermal state (Connors et al. 1980; Laor et al. 1990; Dovciak et al. 2008).

\section{RETURNING RADIATION: QUALITATIVE DISCUSSION}

When returning radiation is included, although little changes in terms of the total observed spectrum, the polarization picture (Fig. 3) changes significantly - in much of the inner disk, the integrated polarization rotates by $90^{\circ}$, even though none of the model's physical parameters has been changed at all!

This effect can be understood qualitatively in very simple fashion (see also Agol \& Krolik (2000)). For most reasonable stellar-mass BH accretion disk models, the opacity in the inner disk is dominated by electron scattering (Shakura \& Sunyaev 1973; Novikov \& Thorne 1973), so returning radiation in the $\sim 1-10 \mathrm{keV}$ energy range should scatter off the disk with negligible absorption. Detailed atmosphere calculations (S. Davis, private communication) show that the photospheric region is strongly scattering dominated for $R \lesssim 50 M$ whenever $L / L_{\mathrm{Edd}} \gtrsim 0.01$. If these calculations were altered to take into account magnetic contributions to vertical support, which should be substantial at these altitudes in this regime (Hirose et al. 2009), absoroption opacity would likely be even weaker (however, when the central mass is much larger, as in an AGN, the inner disk temperature is much lower, giving significantly greater absorptive opacity in the X-ray band). Moreover, the typical photon scatters only once or twice before permanently departing the disk. Its outgoing polarization is therefore very sensitive to the electron scattering cross section's polarization-dependence:

$$
\left(\frac{d \sigma}{d \Omega}\right)_{\mathrm{pol}}=r_{0}^{2}\left|\mathbf{f}_{i} \cdot \mathbf{f}_{f}\right|^{2}
$$

where $r_{0}$ is the classical electron radius. In other words, scattering is strong only when the polarization direction changes little. This is possible for both senses of linear polarization when the change in photon direction is small, but when the scattering is nearly perpendicular, the new photon wave-vector becomes nearly parallel to one of the initial polarization directions. Thus, for nearly-perpendicular scattering, the outgoing light can be close to 100\% polarized.

For observers at high inclination angles, such as in Figure 3 , returning radiation photons initially emitted from the far side of the disk (top of the image) are reflected off the near 
(bottom) side with a relatively small scattering angle, maintaining a moderate horizontal polarization as in Figure 1. On the other hand, photons emitted from the left side of the disk can be bent back to the right side (or vice versa), and then scatter at roughly $90^{\circ}$ to reach the observer, thereby aquiring a large vertical polarization component. Although relatively small in total flux, this latter contribution can dominate the polarization because it is so strongly polarized. A flux component of only $10 \%$, when $100 \%$ polarized, contributes more to the polarized flux than does a $90 \%$ contribution that is only $5 \%$ polarized.

Because high-energy photons from the hotter inner parts of the disk experience stronger gravitational deflection, they are more likely to return to the disk than the low-energy photons emitted at larger radii. We find that, for each value of the spin parameter, there is a characteristic "transition radius," within which the returning radiation dominates and produces net vertical polarization. Outside this point, the direct radiation dominates and produces horizontal polarization. This transition radius is in fact only weakly dependent on $a / M$, ranging from $R_{\text {trans }} \approx 7 M$ for $a / M=0$ to $R_{\text {trans }} \approx 5 M$ for $a / M=0.998$. The location and shape of the observed polarization swing can be used to infer the radial temperature profile near the transition radius (see Sec. 6 below for more details). While the majority of the returning obviously originates from the inner-most disk, we find that most of it also scatters inside $R \lesssim 10 M$ as well (where the atmosphere opacity is completely dominated by electron scattering), due to the additional focusing effects of gravitational lensing.

\section{RETURNING RADIATION: QUANTITATIVE RESULTS}

Polarization maps like that shown in Figure 3 provide a useful picture for understanding the physical origin of the local polarization features, but are less useful in providing quantitative predictions for future observations. Since vertically- and horizontally-polarized photons add up to give zero net polarization, it is not clear from Figure 3 alone what the integrated polarization signal should be. Furthermore, Figure 3 shows only the energy-integrated polarization at each point in the disk, masking the important energy dependence of polarization seen in Figure 2.

In Figures 46 we show the spectral intensity, degree, and angle of polarization as a function of photon energy for a range of $\mathrm{BH}$ spins and inclinations. In each frame, we plot the total flux (solid curves), as well as the direct flux from the disk (dotted curves) and the returning radiation (dashed curves). Beginning with the spectral intensity, we see the standard broad thermal peak characteristic of BHs in the "high soft" state. For BH masses around $10 M_{\odot}$ and luminosities of $\sim 0.1 L_{\mathrm{Edd}}$, the thermal spectrum peaks around $1 \mathrm{keV}$. For

a Novikov-Thorne (NT) emission profile, the direct radiation from a non-spinning BH (peak 
emissivity at radius $R \sim 9 M$ ) dominates over the returning radiation by a factor of roughly 100. As the spin increases and the peak emission region moves closer to the horizon, the relative fraction of observed flux that comes from returning radiation increases to $\sim 5 \%$ for $a / M=0.9$ and $\sim 20 \%$ for $a / M=0.998$. Naturally, the relative contribution from returning radiation increases with energy because the highest energy photons come from the innermost regions of the disk. In fact, for $a / M=0.998$, the returning radiation actually dominates the spectrum above $\sim 10 \mathrm{keV}$.

For all spins, the fraction of returning radiation increases with inclination for two reasons: forward/backward scattering has a larger differential cross-section than right-angle scattering, so returning photons, which characteristically have large angles of incidence, are reflected at large angles from the axis and preferentially reach observers with high inclination; secondly, the direct radiation suffers limb-darkening effects at high inclination due both to the scattering atmosphere and also the diminished solid angle of the disk, while both effects affect the reflected radiation to a lesser degree (see Paper I for a detailed calculation). For the examples shown in Figures 4 6, the fraction of observed flux coming from returning radiation is larger for $i=75^{\circ}$ than $i=45^{\circ}$ by a factor of about two.

At low energies (below the thermal peak), the flux comes predominantly from the outer regions of the disk, where relativistic effects are negligible, so the classic results of Chandrasekhar (1960) are reproduced: horizontal polarization with amplitude of a few percent. The degree of polarization at low energies increases with observer inclination, ranging from $\delta=1 \%$ for $i=45^{\circ}$ to $\delta=3.5 \%$ for $i=75^{\circ}$. Although the free-free absorption opacity in the disk increases below $\sim 1 \mathrm{keV}$, which should reduce the net polarization, we have ignored this effect for two reasons. First, large uncertainties in the vertical structure of the disk make it difficult to arrive at a quantitative prediction for the absorption profile, which is strongly dependent on local temperature and density. Second, most proposed X-ray polarization missions will be sensitive only to photons above $1 \mathrm{keV}$ (Bellazzini et al. 2006), so we choose to focus on this energy range.

Above the thermal peak, we see the relativistic effects described in the previous Sections begin to dominate the polarization signal. The direct radiation (dotted curves) undergoes lensing and beaming, thereby reducing the polarization amplitude and rotating the angle. The polarization properties of the returning radiation (dashed curves) are roughly constant in the $1-10 \mathrm{keV}$ range, but as the relative flux from returning radiation increases, we see the total polarization makes a transition from following the direct behavior to following the returning behavior. In the process of making this transition from horizontal polarization at low energies to vertical polarization at high energies, the degree of polarization goes through a minimum as the two contributions cancel each other. This transition is sharpest for lower 
inclinations, where there is a smaller degree of horizontal polarization to overcome. Yet even for large inclination angles, when the BH spin is high enough, we still see a dramatic transition from horizontal to vertical polarization close to the thermal peak, caused by the enhanced level of returning radiation from the innermost disk.

Due to this dependence on the relative contribution from returning radiation, if the emissivity profile is NT, the energy at which the polarization transition takes place may be a powerful diagnostic of $\mathrm{BH}$ spin. In Figure 7 we plot the polarization degree and angle as a function of energy for a range of spin parameters. In all cases, the $\mathrm{BH}$ mass is $10 M_{\odot}$, $i=75^{\circ}$, and the luminosity is $0.1 L_{\mathrm{Edd}}$ (due to differences in accretion efficiency, we use a fixed luminosity, not mass accretion rate, when comparing different spins). For inclinations of $i=45^{\circ}$ or $60^{\circ}$, this same dependence on spin can be seen by comparing the top and middle rows of Figures 46. With even moderate energy resolution and polarization sensitivity of $\lesssim 1 \%$, observations of BHs in the thermal state should be able to distinguish between Schwarzschild and extremal Kerr with high significance - if one can safely assume the NT surface brightness profile.

However, recent relativistic MHD simulations suggest that, contrary to the NT assumption of zero stress and zero dissipation inside the ISCO, there are in fact significant stress and dissipation in the plunging region, leading to steadily increasing surface brightness (as measured in the local fluid frame) with decreasing radius (Noble et al. 2008). For a simplistic treatment of this flux, we apply a quasi-Newtonian emissivity profile with $\mathcal{F}(R) \sim R^{-3}$ all the way to the horizon, regardless of the BH spin or ISCO location. The local spectral distribution is still given by equations (1-2). The resulting polarization signals are plotted in Figure 8, showing a clear reduction in the sensitivity to spin parameter. While there is still some variation between the different curves, it would likely be impossible to measure $a / M$ with a first-generation X-ray polarization mission (see below, Sec. 66).

The transition energy in the polarization angle is a strong function of the luminosity, which in turn is a function of the temperature of the inner disk. Since the initial polarization and subsequent scattering-induced polarization are independent of frequency at these photon energies, we can think of the luminosity dependence simply as a rescaling of the entire polarization spectrum with inner disk temperature. This scaling is illustrated in Figure 9, which plots the polarization signature for a range of luminosities, in all cases using a NT flux profile with a diluted thermal spectrum and BH mass $10 M_{\odot}$, for $a / M=0$ (solid curves) and $a / M=0.9$ (dashed curves). Since most galactic BHs are observed over a wide range in luminosities, even within the thermal state, multiple polarization observations could give even better constraints on the value of the spin parameter. By reproducing the same polarization at a shifted energy, such a measurement would also help confirm that the features in the 
polarization spectrum are in fact coming from relativistic effects in the inner disk. Again, if the underlying emission profile increases continuously down to the horizon (see Fig. 10), we lose some sensitivity in determining the spin, but gain sensitivity to $L / L_{\text {Edd }}$ because the dependence on that variable sharpens.

\section{PARAMETER ESTIMATION}

As shown in the previous section, spectropolarimetry measurements of BHs in the thermal state will give a powerful new way of measuring $\mathrm{BH}$ spin, if the underlying emission follows a Novikov-Thorne profile with zero dissipation inside of the ISCO. More generally, polarization observations will probe the emissivity profile directly, offering a new way to measure the temperature profile as a function of the geometrized radius $(R / M)$. Well outside the ISCO, conservation laws constrain all models of time-steady disks to follow the NT form; however, nearer the ISCO and throughout the plunging region inside it, more variation is possible. Motivated by global MHD simulations such as those presented in Noble et al. (2008) and Beckwith et al. (2008), we describe the range of possible dissipation profiles by power-laws in radius in the plunging region matched smoothly to the NT profile just outside the ISCO. Figure 11 shows a sample of emission profiles for $a / M=0.9$ and a range of power-law index $\alpha$. In the limit $\alpha \rightarrow-\infty$, we reproduce NT; $\alpha=3$ is the pseudo-Keplerian case shown above in Figures 8 and 10, Also shown for comparison is the dissipation profile calculated by HARM3D for the case of $a / M=0.9$ and a disk aspect ratio $H / R \simeq 0.1$, which corresponds to $\alpha \approx 0.5-1$ (Noble et al. 2008).

To estimate the ability of polarization observations to accurately determine $a / M$ and $\alpha$ for a typical galactic BH binary, we consider two theoretical X-ray polarimeters: a "firstgeneration" instrument with modest energy range $1-10 \mathrm{keV}$, resolution $\Delta E / E \approx 1$, and minimum polarization sensitivity of $\delta \gtrsim 1 \%$ with $1 \sigma$ confidence. A "next-generation" detector is characterized by a broader energy range $0.1-100 \mathrm{keV}$, better resolution $\Delta E / E \approx 0.1$, and ten times the collecting area, giving $\delta \gtrsim 0.3 \%$ for the same source. We then select a few sample "target" models with different emission profiles and generate simulated polarization data for these cases using the ray-tracing code described above. Scanning over a range of model parameters, we calculate a large number of "template" polarization spectra to be matched against the targets.

For each point in parameter space, we calculate the overlap between a template spectrum and the target spectrum by calculating $\chi^{2}$ for the specified detector sensitivity and source luminosity. Rather than attempting to combine the absolute luminosity, distance, detector response function and collecting area into an error estimate for a generic spectro- 
polarimeter, we simply describe the instrument and source together in terms of the minimum error achievable in a measurement of $\delta$ for a single energy bin. For a broad-band detector with constant sensitivity at all energies, the minimum error will correspond to the peak of the observed intensity spectrum, i.e. the bin with the most photon counts. The error in any other bin simply scales like the square root of the counts in that bin. We use for our observables the Stokes parameters $Q_{\nu}$ and $U_{\nu}$, both normalized to the total observed flux $I_{\text {tot }}=\int I_{\nu} d \nu$. For a peak sensitivity (minimum error) of $\delta_{\min }$, the error on each value of $Q_{\nu}$ and $U_{\nu}$ is given by

$$
\Delta Q_{\nu}=\Delta U_{\nu}=\delta_{\min } I_{\nu} \sqrt{\frac{I_{\mathrm{peak}}}{I_{\nu}}}
$$

With the target values of $Q_{\nu}$ and $U_{\nu}$ and their associated measurement errors at each energy, it is straightforward to calculate the $\chi^{2}$ fit for the match between the target "observation" and any given template spectrum. By generating multiple templates to cover the parameter space, we can estimate the significance with which the model parameters can be determined from future data.

In practice, this search through parameter space would be prohibitively expensive computationally if each template were constructed using the full Monte Carlo calculation (typically $\sim 10^{8}$ rays traced per run). Fortunately, we are able to utilize transfer functions similar to those used in calculating the relativistic effects on broad-band continuum spectra (e.g. Davis et al. (2005)). The full details are described in Paper I, but a short summary of the method is as follows: for each value of the spin parameter (roughly 20 points spaced evenly in $\log (1-a / M)$ ), a single complete Monte Carlo simulation is carried out as above, with the emission spectrum at each radius given by a delta-function in energy. The initial polarization is still given by a scattering-dominated atmosphere, and the returning radiation is treated as before, typically adding a strong vertical component to the observed polarization. When each ray reaches an observer at infinity, the Stokes parameters $I_{\nu}, Q_{\nu}$, and $U_{\nu}$ are recorded, each looking essentially like a delta function shifted in energy and amplitude by the relativistic effects of the ray-tracing.

Sorting all the rays by observer inclination angle, we construct a transfer function $g(a / M)$ that takes the input emission profile $I_{\nu}^{\mathrm{em}}(R)$ and generates the observed polarization in terms of $I_{\nu}^{\mathrm{obs}}(i), Q_{\nu}^{\mathrm{obs}}(i)$, and $U_{\nu}^{\mathrm{obs}}(i)$. For a given spin, a single transfer function can be used to generate polarization spectra for any set of model parameters $M, L / L_{\mathrm{Edd}}$, and $\alpha$, all of which combine to give $I_{\nu}^{\mathrm{em}}(R)$. The transfer function can also be used to calculate the transition radius $R_{\text {trans }}$ described above in Section 4. For emission profiles with steep gradients around $R_{\text {trans }}$ (as would be produced by large values of $a / M$ or $\alpha$; see Figs. 8 and 10), the observed polarization spectrum displays a sharp swing around the photon energy corresponding to the disk temperature at that radius: $E_{\text {trans }} \approx 3 T\left(R_{\text {trans }}\right)$. On the other 
hand, moderate spin systems with NT emission profiles have a shallow temperature gradient around $R_{\text {trans }}$, giving a broad swing in the polarization angle (Figs. 7 and 9), or none at all in low-spin cases where there is simply not enough emission from inside $R_{\text {trans }}$ to overcome the horizontal contribution from emission outside of $R_{\text {trans }}$.

For reasonably high resolution spectra, we find the transfer function method gives a speed-up factor of better than 10,000 compared to the direct Monte Carlo calculation. This method is particularly straightforward in the thermal state, where the scattering cross sections and polarization transport are essentially independent of energy. However, even when including inverse-Compton coronal scattering, the transfer function method is still applicable, with the added dimension that the transfer function is dependent on the seed photon energy and accretion geometry (again, see Paper I for details).

In Figure 12, we show quality-of-fit contours for matching the target parameters of three different models: two NT radial profiles with $a / M=0$ and $a / M=0.998$, and one case with additional emissivity in the plunging region with $\alpha=1$ and $a / M=0.97$. All three cases have $M=10 M_{\odot}, L / L_{\mathrm{Edd}}=0.1$, and $i=75^{\circ}$. The top row of figures corresponds to cases where the disk inclination and Eddington-scaled luminosity (i.e. the $\mathrm{BH}$ mass and distance) are known a priori, and the target data is fit by scanning over $a$ and $\alpha$. The middle row repeats this calculation, but assumes prior knowledge only of the disk inclination, and then for each value of $a$ and $\alpha$ minimizes $\chi^{2}$ over $L / L_{\text {Edd }}$. Finally, the bottom row assumes no prior knowledge about the system and minimizes $\chi^{2}$ with respect to $L / L_{\text {Edd }}$ and inclination $i$. In each frame, the target parameters are marked with an ' $\mathrm{X}$ ' and the contours show confidence intervals in significance units: $\leq 1 \sigma$ (white), $2 \sigma$ (blue), $3 \sigma$ (purple), $4 \sigma$ (red), $5 \sigma$ (orange), and $>5 \sigma$ (yellow). Here $\sigma$ is given by a standard $\chi^{2}$ distribution: $\sigma=\sqrt{2 \nu}$, with $\nu$ the number of data points minus the number of free parameters. For large values of $\nu$, these confidence intervals correspond very closely to those of a normal distribution: (68\%, $95.5 \%, 99.7 \%$, etc.).

As seen in Figure 9, the Schwarzschild case closely resembles the Chandrasekhar limit of horizontal polarization with amplitude of a few percent. When the emission cuts off at the ISCO, relativistic effects play a much smaller role in rotating the polarization of the direct radiation. Furthermore, the fraction of returning radiation is much smaller, minimizing the contribution from vertically polarized photons. The only way to generate such a polarization spectum is by producing the observed flux at large radii, from where the photons can propagate through nearly flat space to the distant observer, arriving with their polarization essentially unchanged. Thus if the observed polarization is relatively strong ( $\gtrsim 1 \%$ ), horizontally oriented, and constant with energy, then the emission likely comes from large radii, in turn constraining the spin to be relatively small and strongly limiting the amount 
of dissipation inside the ISCO. This is evident from the upper-left frame in Figure 12, where a polarization observation of a Schwarzschild BH with a NT dissipation profile rules out the possibility of a high value for $a / M$ or $\alpha$.

On the other hand, when matching the polarization from a near-maximal spin BH with $a / M=0.998$ (upper-right frame of Fig. 12), any system with a NT emission profile and spin $a / M \lesssim 0.97$ is ruled out at the $3 \sigma$ confidence level. Yet as described above in Section 5 , a non-spinning $\mathrm{BH}$ can appear to be rapidly spinning if there is significant emission inside the ISCO. Additionally, since the plunging region is so small for large values of $a / M$, templates with a range of $\alpha$ (i.e. the emission profile inside the ISCO) are nearly degenerate, as seen in Figure 8. These systems are characterized by a sharp transition in the polarization: it changes from strong, horizontal polarization at low energies to strong, vertical polarization above the thermal peak.

For intermediate cases with moderately high spin and $\alpha[(0.9 \lesssim a / M \lesssim 0.99) ;(-2 \lesssim$ $\alpha \lesssim 2)$ ], there is a broad minimum in the polarization amplitude between 1 and $10 \mathrm{keV}$ (see, e.g., the red and orange curves in Fig. 7). If the inclination is known to be relatively high, as in our test cases, then this broad minimum can still constrain $a / M$ and $\alpha$ (or at least some combination of the two parameters) reasonably well, as seen in the upper-middle plot of Figure 12. However, if we have no prior knowledge of the disk inclination, we could reproduce such a feature by assuming a low-inclination system, whose light would have little or no polarization near the thermal peak regardless of the emission profile. Therefore, as shown in the lower-middle panel of Figure 12, when minimizing $\chi^{2}$ over all model parameters, it is always possible to get a decent fit to an unpolarized source, and the emission profile is poorly constrained. At the same time, if a non-zero polarization is detected, it can give a measure not only of the emission profile, but also of the disk inclination.

There are also a number of $\mathrm{BH}$ binary systems for which the inclination is known relatively well, but the $\mathrm{BH}$ mass $M$ and/or distance $D$ are not known. This is the situation illustrated in the middle row of Figure 12. As those panels show, substantial constraining power is retained even without knowledge of $L / L_{\mathrm{Edd}}$ (and as the bottom panels show, even when the inclination angle is also unknown, spectropolarimetry still provides some limit on the possible range of $\alpha$ and $a / M)$. The reason is that with a single spectropolarimetry observation, we can measure, using the shape and energy of the polarization transition, the temperature profile $T(r)$ in the inner disk as a function of radius in geometric units $r \equiv R / M$. From this temperature profile, we can constrain the system's ratio of bolometric luminosity to mass through the relation:

$$
L \propto M^{2} \int r T(r)^{4} d r .
$$

Using the net flux gives $L / D^{2}$, so that we have two constraint equations for three unknowns. 
If either the $\mathrm{BH}$ mass or the distance can be measured by some other means, all three variables $(L, M$, and $D)$ will be known.

Viewed in this way, spectropolarimetry measurements can be seen to have significant advantages relative to pure spectral measurements. When the inclination, mass, and distance are all known, detailed continuum-fitting can be used to measure the temperature profile in the inner disk, giving constraints on $\alpha$ and $a / M$ that are comparable to what is shown in the top row of Figure 12. However, when one or more of these quantities (inclination, distance, or mass) is not known, the continuum-fitting method becomes less able to constrain the radial emission profile and thus the $\mathrm{BH}$ spin.

At the qualitative level, one can understand the greater power of spectropolarimetry as simply the result of having more observables: $\delta\left(E_{\text {obs }}\right)$ and $\psi\left(E_{\text {obs }}\right)$ in addition to $F\left(E_{\text {obs }}\right)$, giving more constraints on the model parameters. However, there are more specific reasons as well. On the one hand, inferring parameters from continuum spectra can be very ambiguous because there are multiple ways to reproduce an observed spectrum by varying $M, D, i$, and $L / L_{\mathrm{Edd}}$. On the other hand, especially for high-inclination systems, the polarization tends to constrain individual parameters more directly: for example, its magnitude at low energies (where relativistic effects are weak) directly relates to the inclination. At higher energies, the distinctive swing in polarization angle is a direct probe of extreme gravitational lensing and returning radiation, giving a sensitive indicator of strongly relativistic effects in the inner disk.

Not surprisingly, a next-generation polarimeter with roughly a factor of ten improvement in energy bandwidth, resolution, and collecting area can do a much better job at measuring all these system parameters. Figure 13 shows the same confidence contours as Figure 12 , now for the next-generation detector. Almost all degeneracy has been removed, and even when we have no prior knowledge of the binary parameters, $a / M, \alpha, i, L / M^{2}$, and $M / D$ can be determined with high precision. For the three cases considered in the bottom row of Figure 13, the inclination is recovered within $\sim 5^{\circ}$ and the accretion rate within $\sim 20 \%$ :

$$
70^{\circ} \lesssim i \lesssim 80^{\circ}
$$

and

$$
0.08 \lesssim\left(\frac{L}{L_{\mathrm{Edd}}}\right)\left(\frac{10 M_{\odot}}{M}\right) \lesssim 0.12 .
$$

In fact, at these levels of polarization sensitivity, the ability to measure the intrinsic properties of the system likely becomes more dependent on the accuracy of the underlying emission model, rather than on the signal-to-noise of the observation. For example, the simplified form of the emission spectrum may be modified by a more careful treatment of 
the disk atmosphere (Davis et al. 2005). Additionally, the low-energy polarization may be strongly modified or reduced by absorption in the disk (Laor et al. 1990), Faraday rotation as the photons pass through turbulent magnetic fields (S. Davis, private communication), and even a small amount of inverse-Compton scattering that is sometimes seen in the thermal state.

\section{DISCUSSION}

We have presented here the first results of a new Monte Carlo ray-tracing code for calculating the X-ray polarization from black holes. This code, described in detail in Paper $\mathrm{I}$, is most notable for its emitter-to-observer paradigm of radiation transport, which allows for the inclusion of returning radiation and electron scattering in a completely general accretion geometry. In this paper we focus on polarization signatures of the thermal state in stellarmass black holes, a condition in which the disk is optically thick and geometrically thin, and its opacity is scattering-dominated. The emitted radiation has a diluted thermal spectrum and is weakly polarized parallel to the disk surface. The integrated polarization spectrum seen by a distant observer contains distinct energy-dependent features, as the high-energy photons from the inner disk are modified by relativistic effects such as Doppler boosting and gravitational lensing near the $\mathrm{BH}$.

For radiation originating very close to the $\mathrm{BH}$, the most important relativistic effect is the strong gravitational lensing that causes the photons to get bent back onto the disk and scatter towards the observer. This scattering can induce very high levels of polarization, especially at large inclination angles, and leads to a distinct transition in the polarization angle from horizontal at low energies to vertical above the thermal peak. Observing such a swing in the polarization angle would give the most direct evidence to date for the extreme relativistic light bending predicted around black holes. Furthermore, by measuring the location and shape of this transition, we will be able to constrain the temperature profile of the inner disk. If we assume a NT disk with zero emission from inside the ISCO, the polarization transition energy gives a direct measurement of the BH spin (see Fig. 7). Alternatively, if we relax this assumption, the polarization can constrain models for the dissipation profile and provide compelling evidence for strong-field gravitational effects.

For BH systems where the mass, inclination, and distance are known from other observations, polarization measurements would be comparable in power to the continuum fitting method (Gierliński et al. 2001; Davis et al. 2006; Shafee et al. 2006) for the purpose of inferring the emissivity profile of the inner disk. However, when we lack knowledge of any one of these priors, polarization provides a significantly stronger tool than continuum fitting for 
constraining both the shape of the emissivity profile and the unknown parameter(s).

In this paper, we have presented the first results from our new black hole X-ray polarization analysis code, corresponding to thermal emission from a geometrically thin accretion disk. In future work, we will extend its application to cases in which a hot corona partially covers a cooler disk (as may be the case in AGN) and to other accretion geometries, appropriate to other spectral states of Galactic black hole binaries. 


\section{REFERENCES}

Agol, E., \& Krolik, J. H. 2000, ApJ, 528, 161

Beckwith, K., Hawley, J.F. \& Krolik, J.H. 2008, MNRAS 390, 21

Bellazzini, R., et al. 2006, Nucl. Instr. Meth., A560, 425

Black, J. K., et al. 2003, Nucl. Instr. Meth., 513, 639

Carter, B. 1968, Phys. Rev., 174, 1559

Chandrasekhar, S. 1960. Radiative Transfer, Dover, New York

Connors, P. A., \& Stark, R. F. 1980, Nature, 269, 128

Connors, P. A., Piran, T., \& Stark, R. F. 1980, ApJ, 235, 224

Costa, E., et al. 2008, Proc. SPIE, vol. 7011-15, arXiv:0810.2700

Cunningham, C. T. 1976, ApJ, 208, 534

Davis, S.W., Blaes, O.M., Hubeny, I. \& Turner, N.J. 2005, ApJ, 621, 372

Davis, S. W., Done, C., \& Blaes, O. M. 2006, ApJ, 647, 525

Dovciak, M., Karas, V., \& Matt, G. 2004, MNRAS, 355, 1005

Dovciak, M., Muleri, F., Goodmann, R. W., Karas, V., \& Matt, G. 2008, MNRAS submitted, arXiv:0809.0418

Gierliński, M., Maciołek-Niedźwiecki, A. \& Ebisawa, K. 2001, MNRAS 325, 1253

Hirose, S., Krolik, J. H., \& Blaes, O. 2009, ApJ, 691, 16

Jahoda, K., Black, K., Deines-Jones, P., Hill, J. E., Kallman, T., Strohmayer, T., \& Swank, J. 2007, [arXiv:0701090]

Laor, A., Netzer, H., \& Piran, T. 1990, MNRAS, 242, 560

Li, L.-X., Narayan, R., \& McClintock, J. E. 2008, ApJ submitted, arXiv:0809.0866]

Matt, G., Fabian, A. C., \& Ross, R. R. 1993, MNRAS, 264, 839

Noble, S. C., Krolik, J. H., \& Hawley, J. F. 2008, ApJ submitted, arXiv:0808.3140] 
Novikov, I. D., \& Thorne, K. S. 1973, in Black Holes, ed. C. DeWitt \& B. S. DeWitt (New York: Gordon and Breach)

Remillard, R. A., \& McClintock, J. E. 2006, ARA\& A, 44, 49

Rybicki, G. B., \& Lightman, A. P. 1979, Radiative Processes in Astrophysics (New York: Wiley-Interscience)

Schnittman, J. D. 2009, in preparation (Paper I)

Shafee, R., McClintock, J. E., Narayan, R., Davis, S. W., Li, L.-X., Remillard, R. A. 2006, ApJ, 636, L113

Shakura, N. I., \& Sunyaev, R. A. 1973, A\&A, 24, 337

Shimura, T., \& Takahara, F. 1995, ApJ, 445, 780

Stark, R. F., \& Connors, P. A. 1977, Nature, 266, 429

Walker, M., \& Penrose, R. 1970, Commun. Math. Phys., 18, 265 
Fig. 1.- Ray-traced image of direct radiation from a thermal disk. The observer is located at an inclination of $75^{\circ}$ relative to the $\mathrm{BH}$ and disk rotation axis, with the gas on the left side of the disk moving towards the observer, which causes the characteristic increase in intensity due to relativistic beaming. The black hole has spin $a / M=0.9$, mass $M=10 M \odot$, and is accreting at $10 \%$ of the Eddington limit with a Novikov-Thorne zero-stress emissivity profile, giving peak temperatures around $1 \mathrm{keV}$. The observed intensity is color-coded on a logarithmic scale and the energy-integrated polarization vectors are projected onto the image plane with lengths proportional to the degree of polarization.

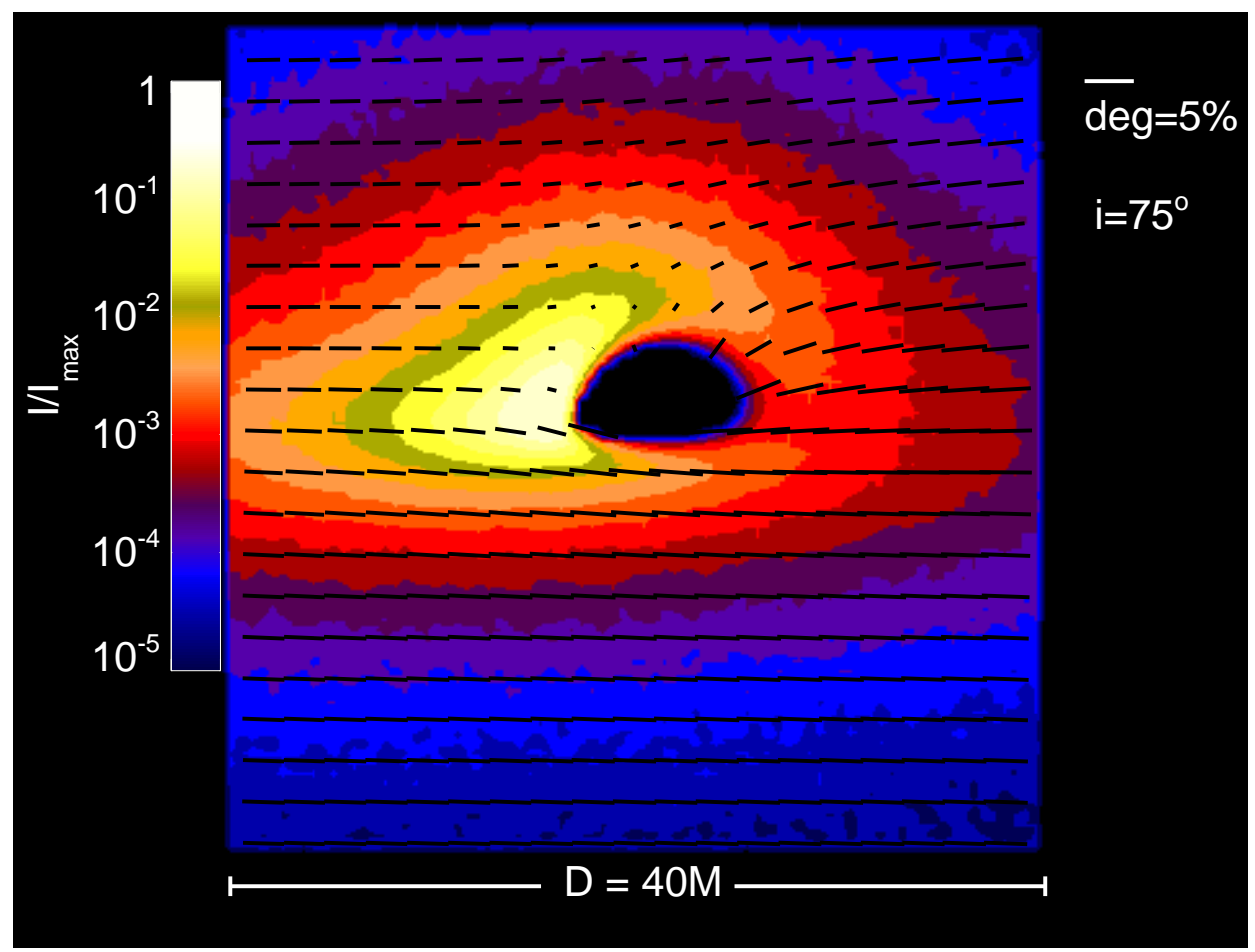


Fig. 2.- Polarization degree and angle from a thermal disk as a function of observed photon energy, including only direct radiation. The BH parameters are as in Fig. 11(dashed curves) and also include identical calculations corresponding to a non-spinning BH (solid curves). The angle of polarization is measured with respect to the horizontal axis in the image plane with point symmetry through the origin: $\psi=\psi-180^{\circ}$.
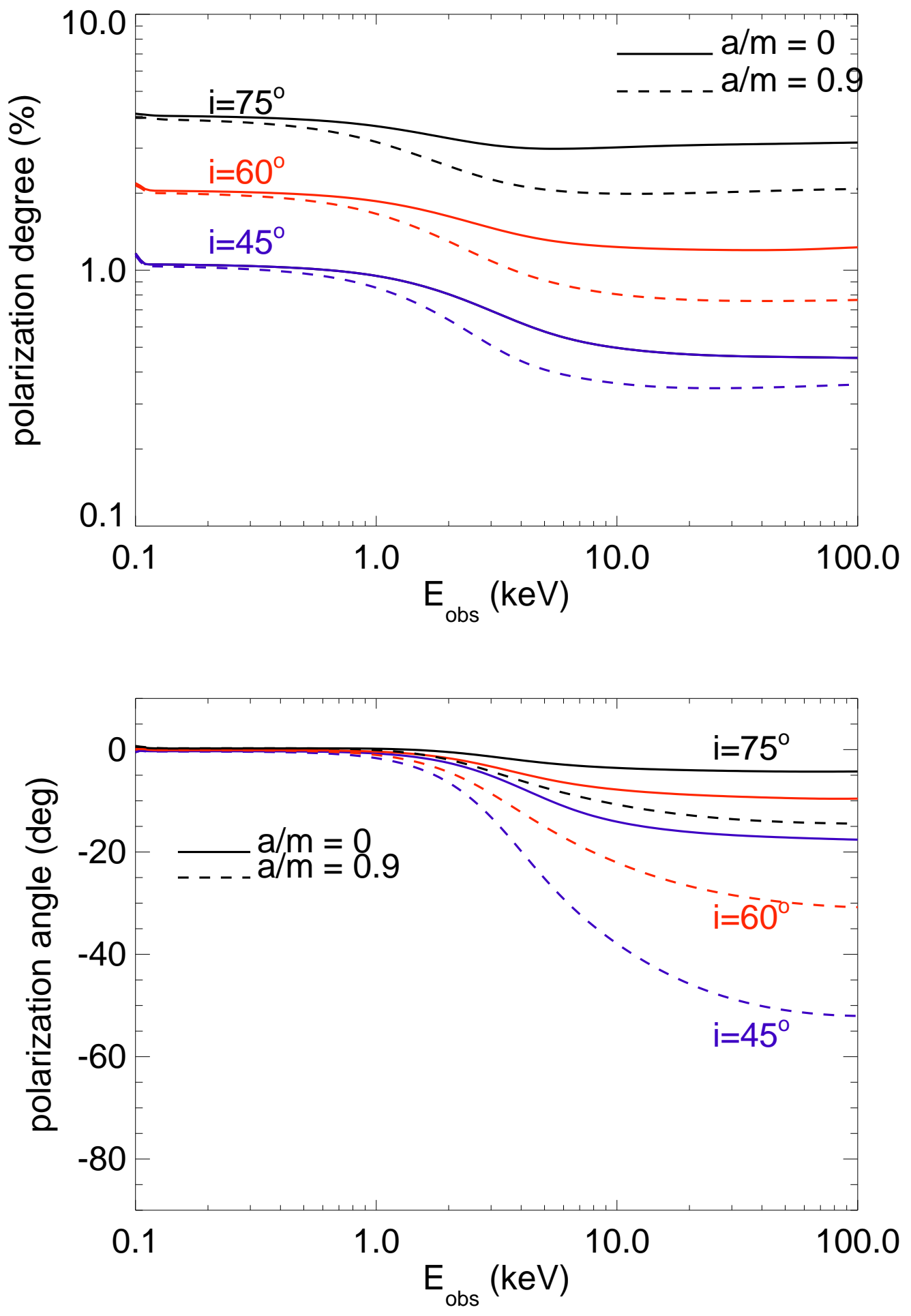
Fig. 3.- Ray-traced image of radiation from a thermal disk, as in Fig. 1, but here including returning radiation. Photons emitted from the inner disk get bent by the $\mathrm{BH}$ and scatter off the opposite side of the disk towards the distant observer.

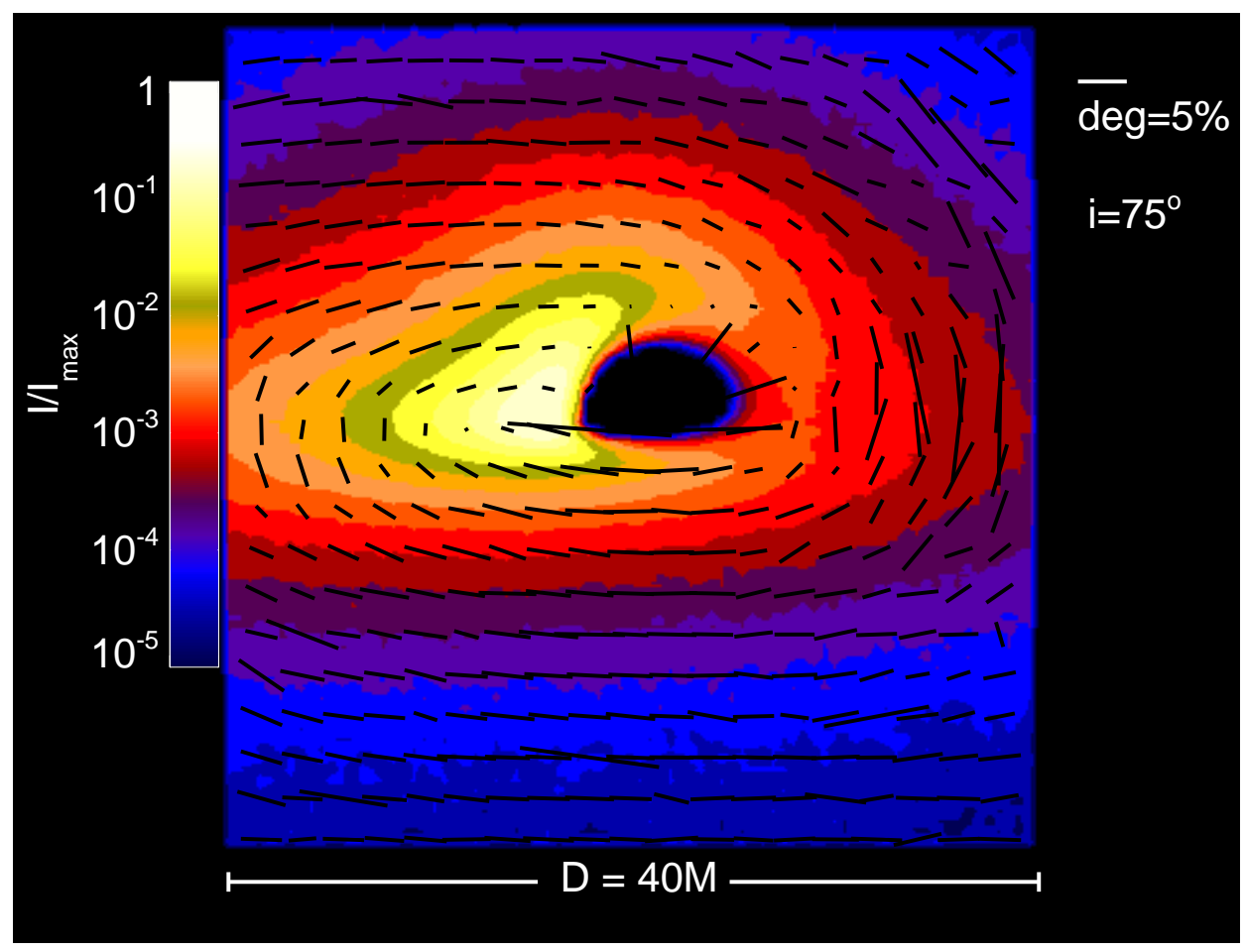


Fig. 4.- Intensity spectrum and polarization degree and angle from a thermal disk, including direct and returning radiation, for a $\mathrm{BH}$ with $a / M=0, M=10 M_{\odot}, L / L_{\mathrm{Edd}}=0.1$, and a Novikov-Thorne emission profile. In the left column we plot the observed flux, in the middle the degree of polarization, and on the right the angle of polarization. In each plot, the flux is divided into contributions from the direct (dotted curves), reflected (dashed curves), and total (solid curves). From top to bottom, the observer inclination is $45^{\circ}, 60^{\circ}$, and $75^{\circ}$.
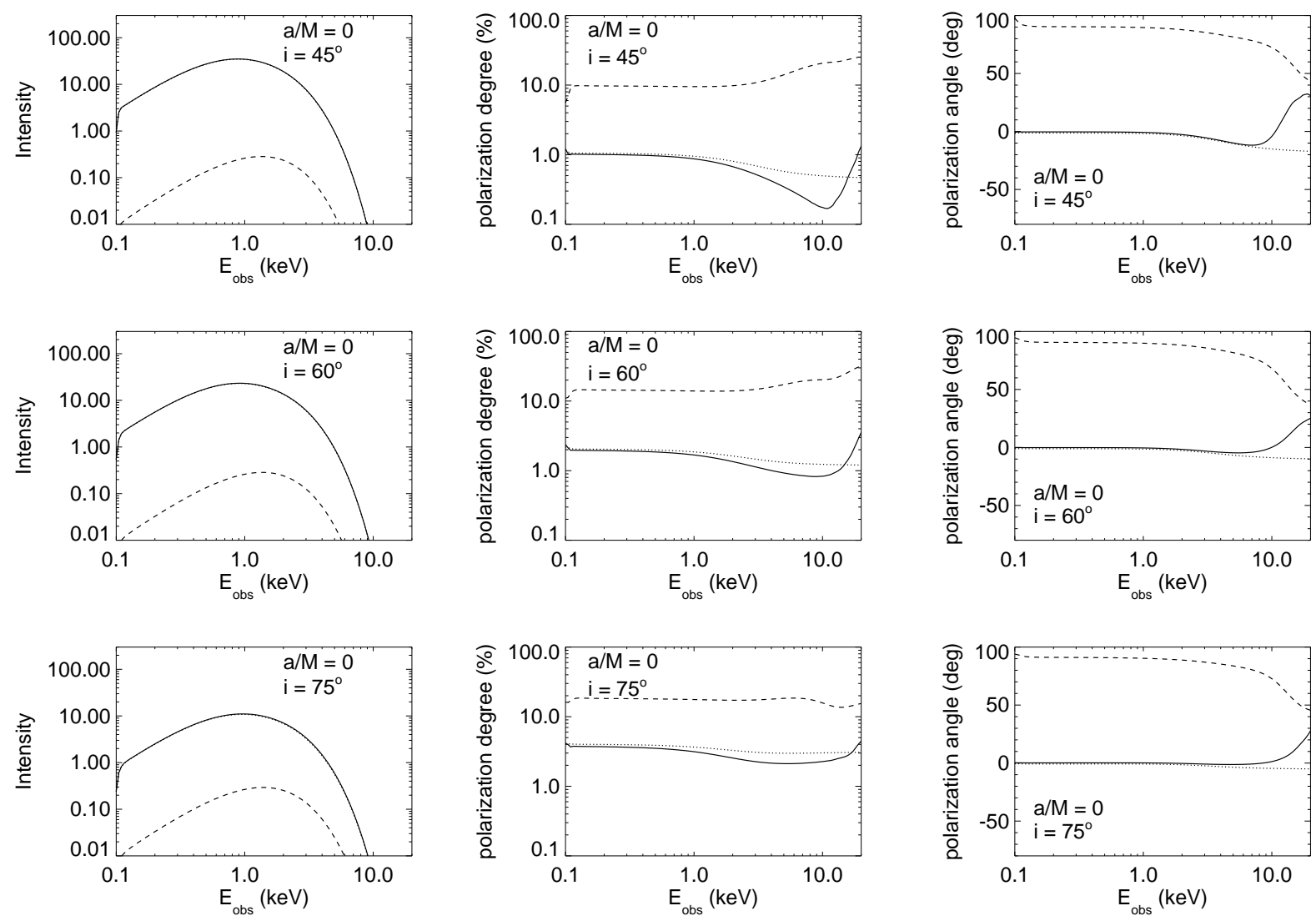
Fig. 5.- Intensity spectrum and polarization degree and angle from a thermal disk, as in Figure 4, but for a Kerr BH with $a / M=0.9$.
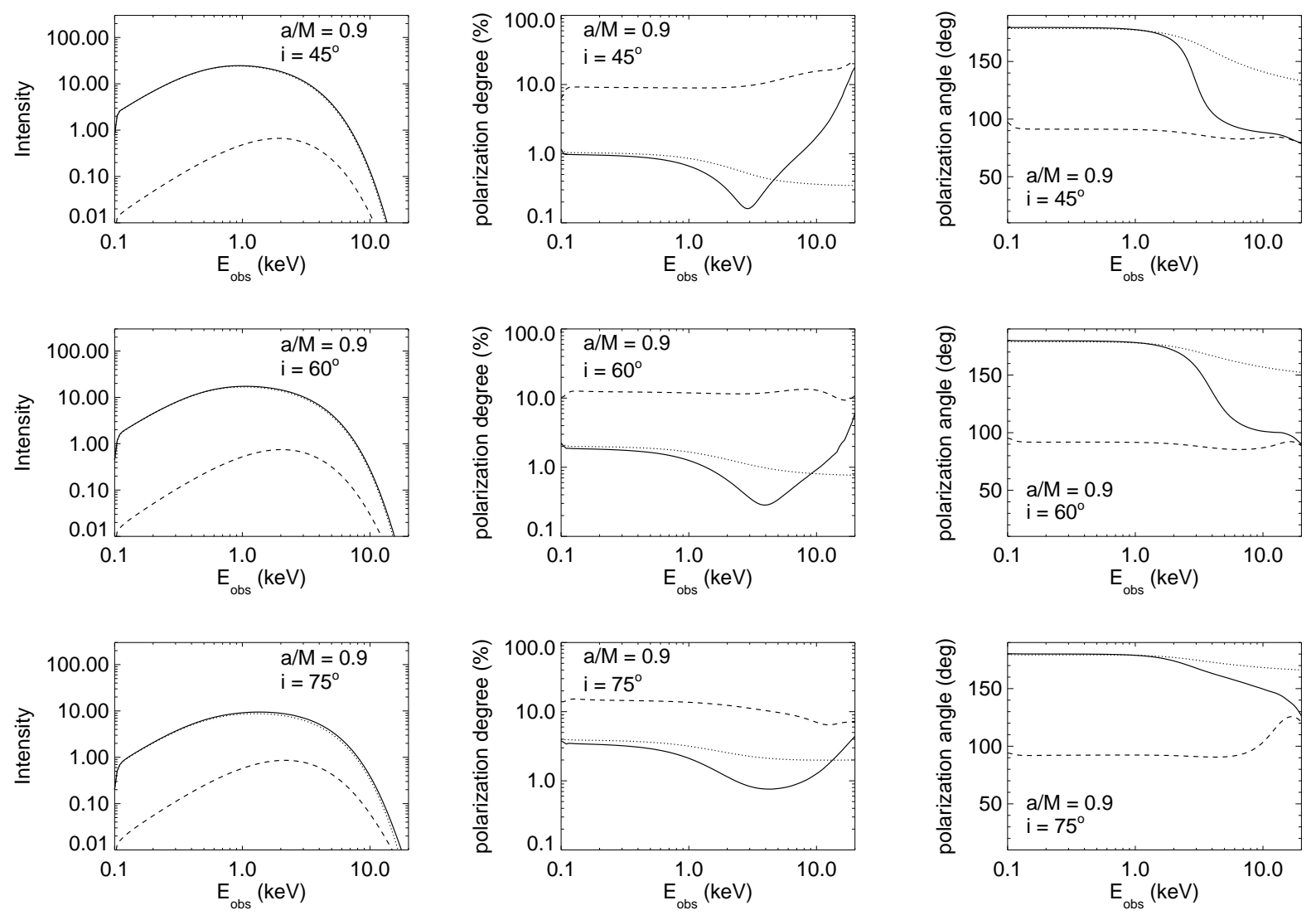
Fig. 6.- Intensity spectrum and polarization degree and angle from a thermal disk, as in Figure 4, but for a Kerr BH with $a / M=0.998$.
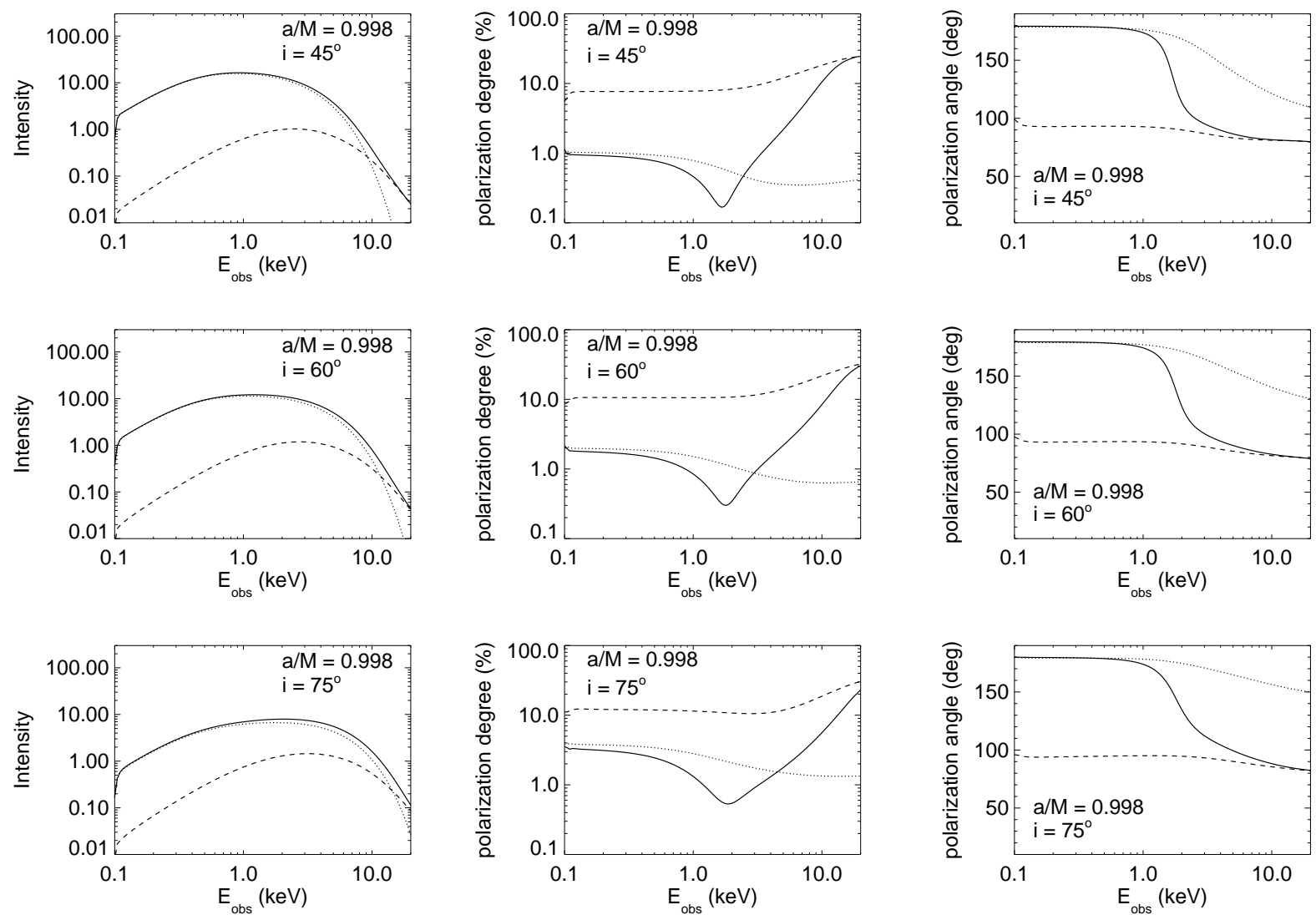
Fig. 7.- Polarization degree and angle for a range of $\mathrm{BH}$ spin parameters. All systems have inclination $i=75^{\circ}, \mathrm{BH}$ mass $10 M_{\odot}$, luminosity $L / L_{\mathrm{Edd}}=0.1$, and Novikov-Thorne radial emission profiles.
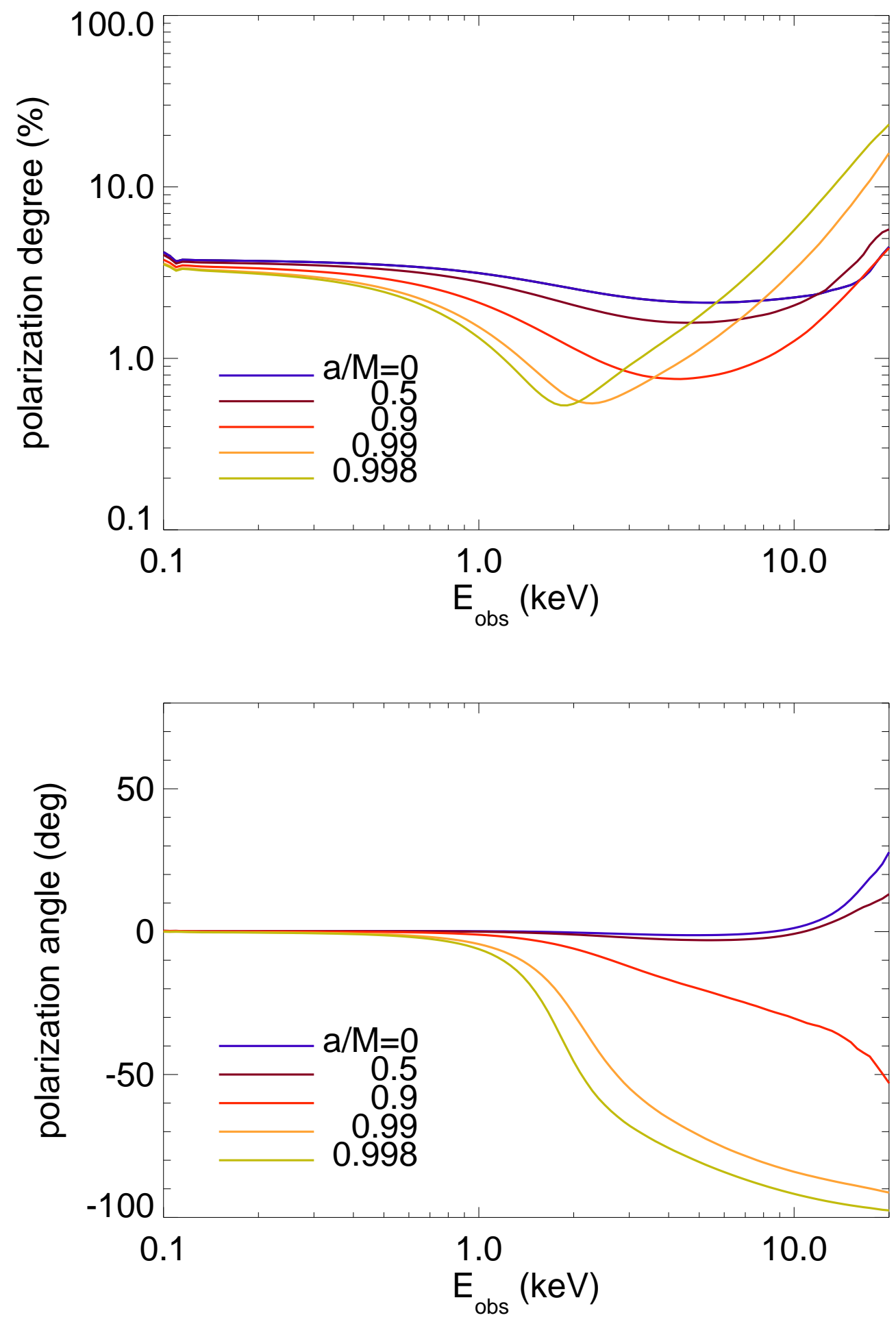
Fig. 8. - Same as Figure 7 but for a power-law emissivity profile $\mathcal{F}(R) \sim R^{-3}$ all the way to the horizon.
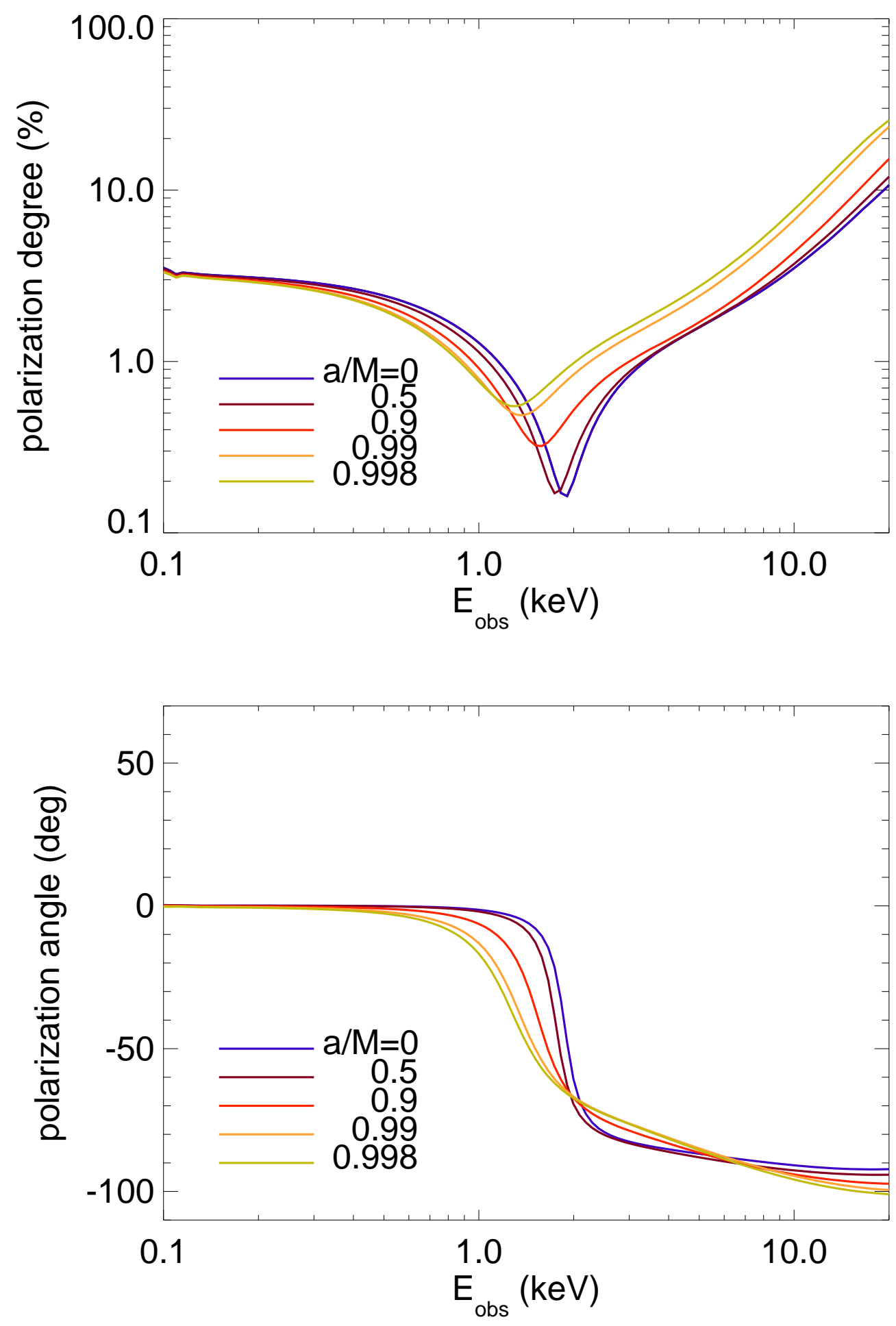
Fig. 9.- Polarization degree and angle for a range of luminosities for $a / M=0$ (solid curves) and $a / M=0.9$ (dashed curves). All systems have inclination $i=75^{\circ}, \mathrm{BH}$ mass $10 M_{\odot}$, and Novikov-Thorne radial emission profiles.
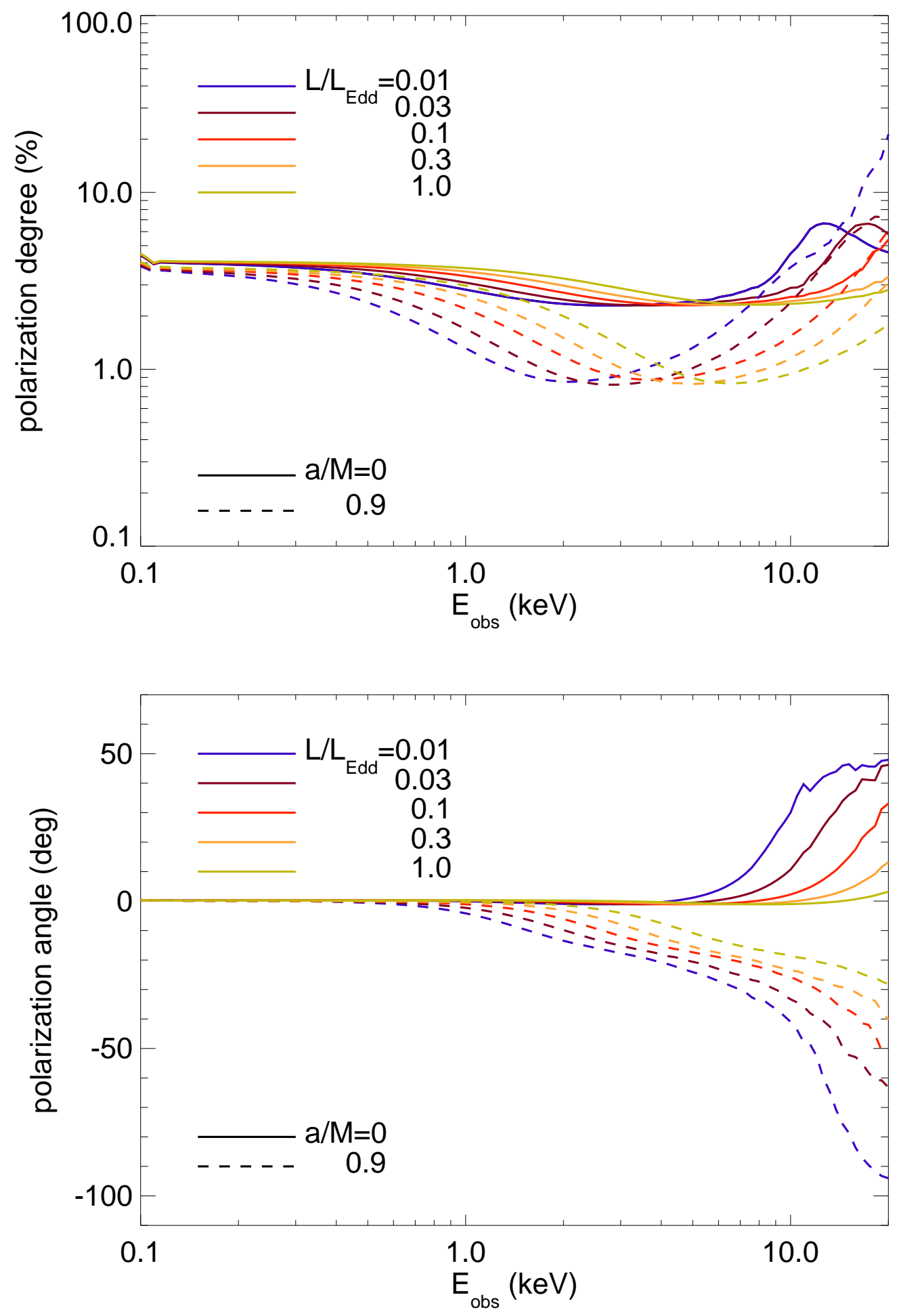
Fig. 10. - Same as Figure 9 but for a power-law emissivity profile $\mathcal{F}(R) \sim R^{-3}$ all the way to the horizon.
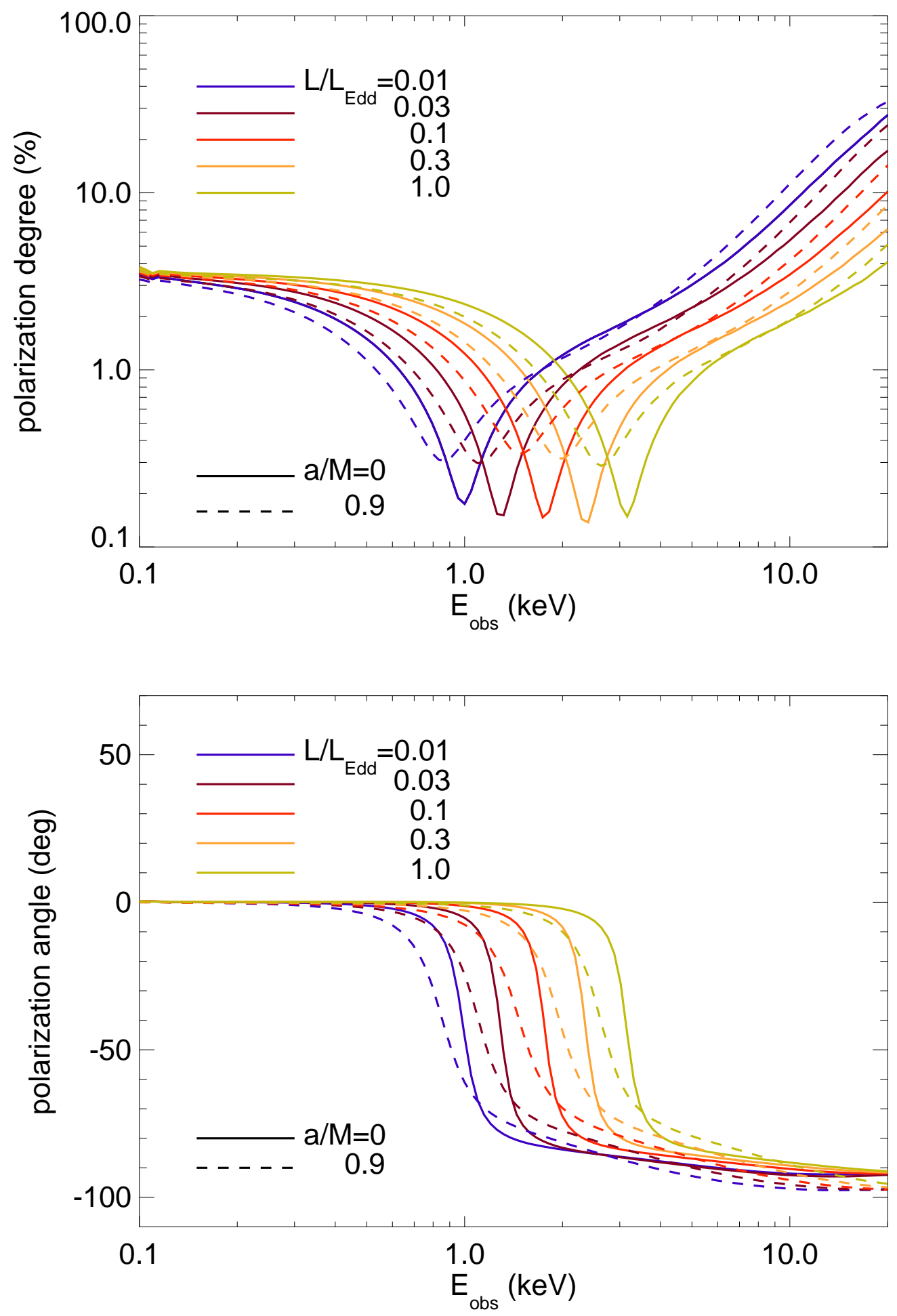
Fig. 11. - Local flux (as measured in the fluid frame) versus radius for $a / M=0.9$. In the plunging region, the flux is parameterized by a power law with index $\alpha$, normalized to match the NT profile (solid curve) just outside the ISCO. Also shown is the emissivity calculated by HARM3D (dotted curve), a 3-dimensional global MHD code in full relativity (Noble et al. 2008).

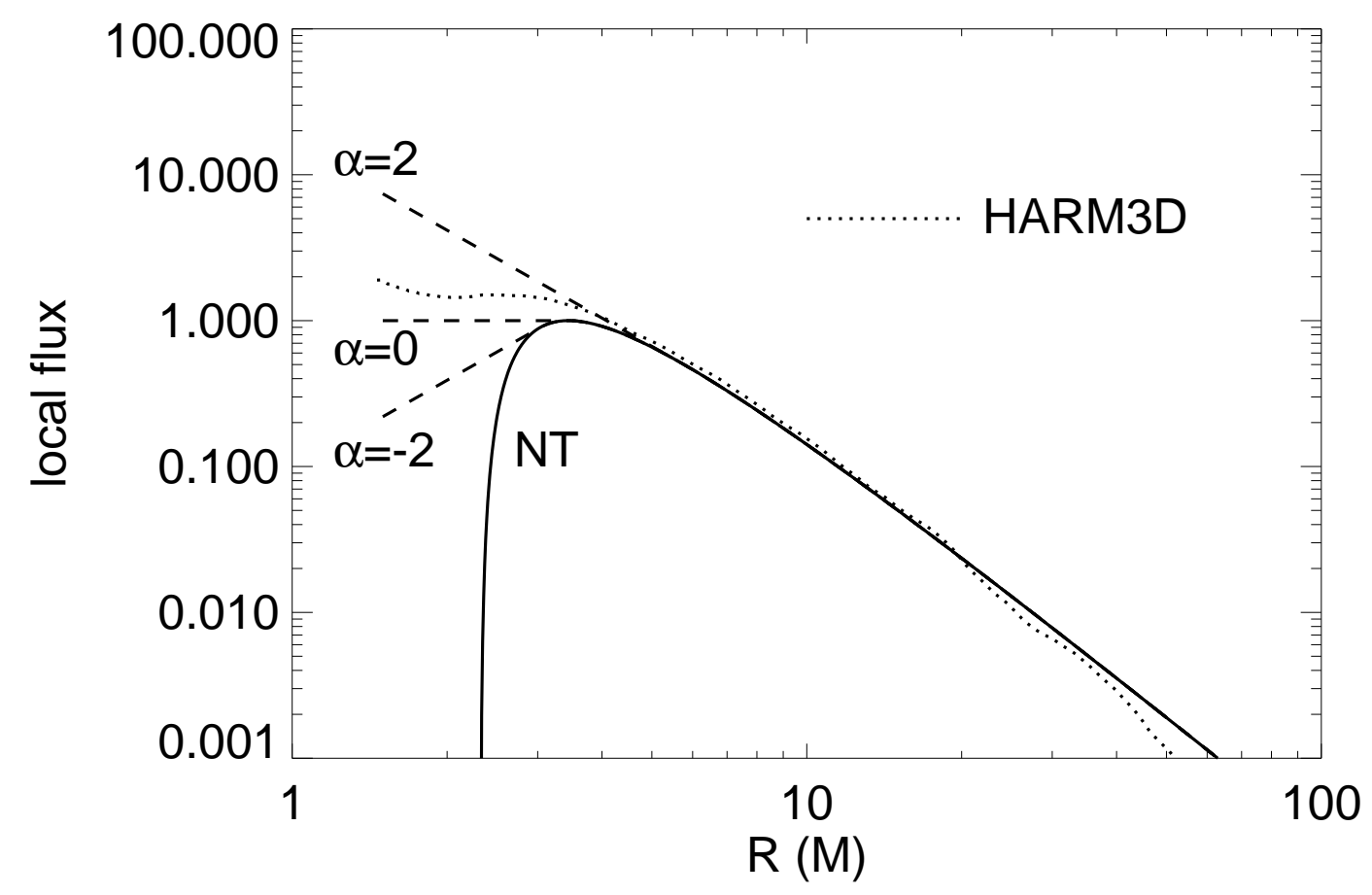


Fig. 12.- Contour plots of fit quality in matching emissivity profiles to simulated data for a first-generation X-ray polarimeter. The left column corresponds to simulated data from a non-spinning Schwarzschild BH with a NT emissivity profile $(\alpha=-\infty)$, the center column has $a / M=0.97$ and $\alpha=1$, and the right column has $a / M=0.998$ and $\alpha=-\infty$. The top row shows the fit quality regions assuming the luminosity in Eddington units and the disk inclination are both known; the middle row assumes we know the disk inclination but not the accretion rate, and the bottom row assumes we know neither. The color coding is $\leq 1 \sigma$ (white), $2 \sigma$ (blue), $3 \sigma$ (purple), $4 \sigma$ (red), $5 \sigma$ (orange), and $>5 \sigma$ (yellow), where $\sigma$ is the variance of the $\chi^{2}(\nu)$ distribution, as defined in the text.
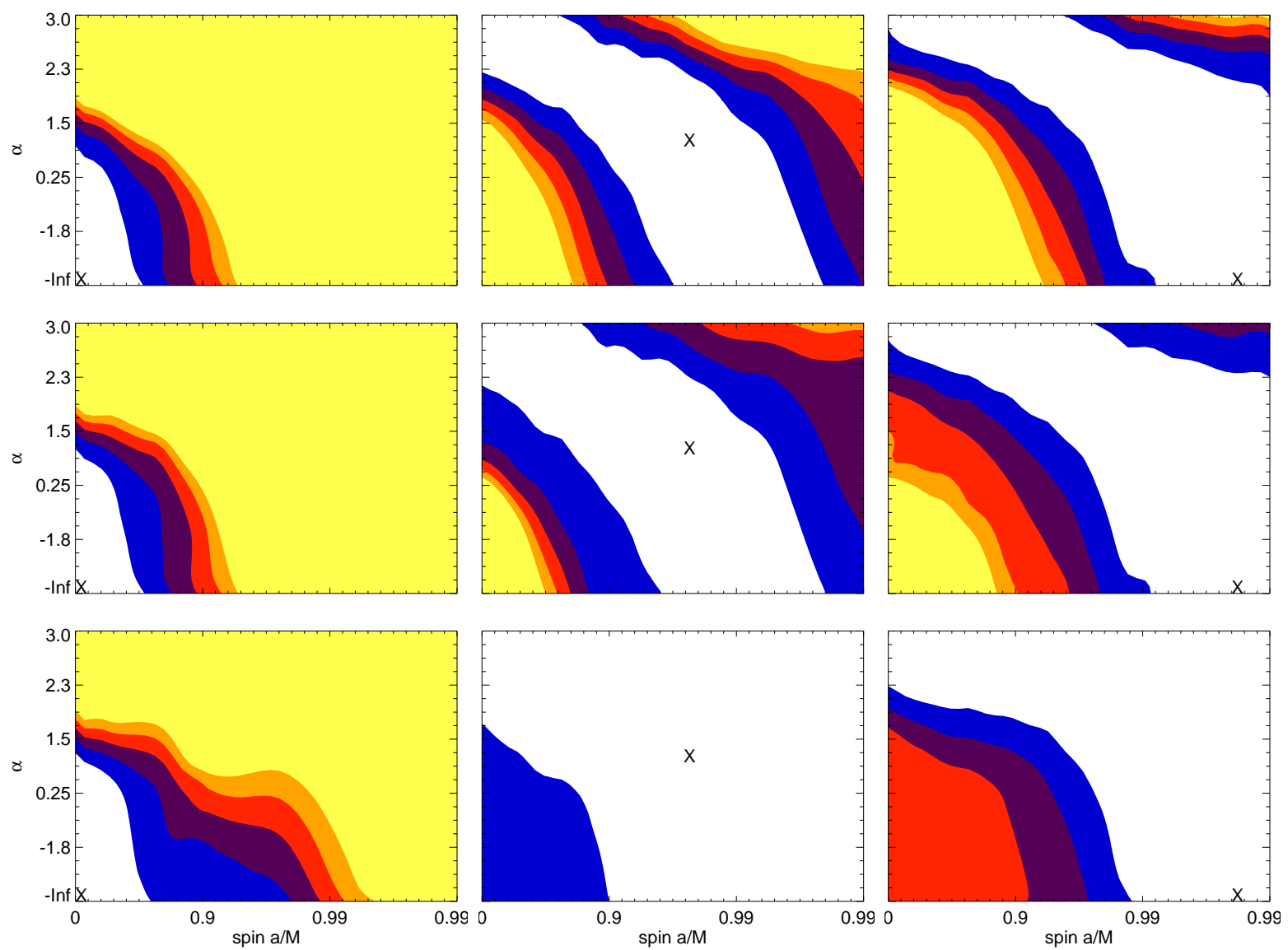
Fig. 13.- Contour plots of fit quality in matching emissivity profiles to simulated data for a next-generation X-ray polarimeter with broader energy range and larger collecting area. The different frames and their color codes are the same as in Figure 12.
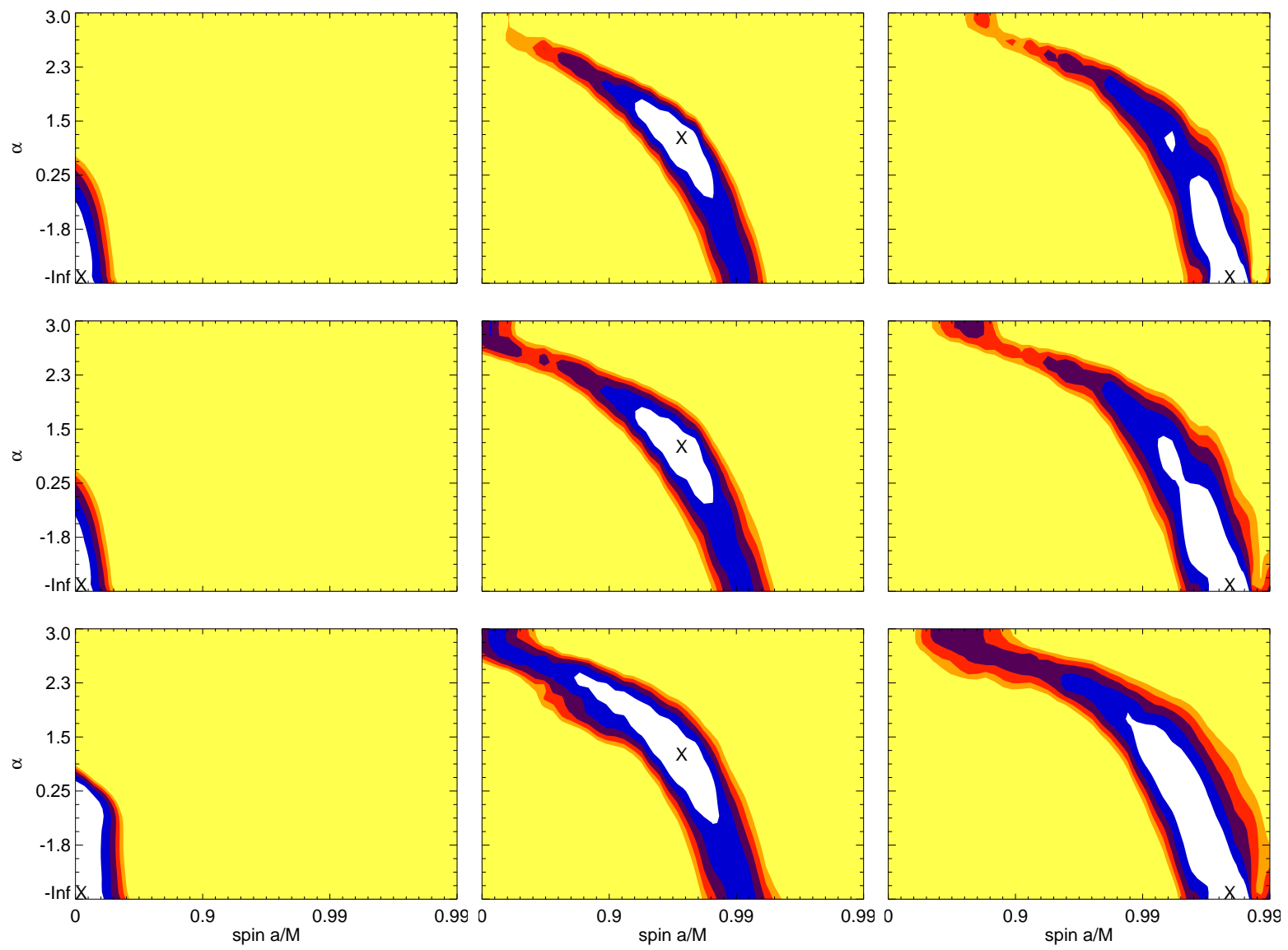